\title{
Mythos, Religion, Gender: Perspektiven auf die Alte Welt
}

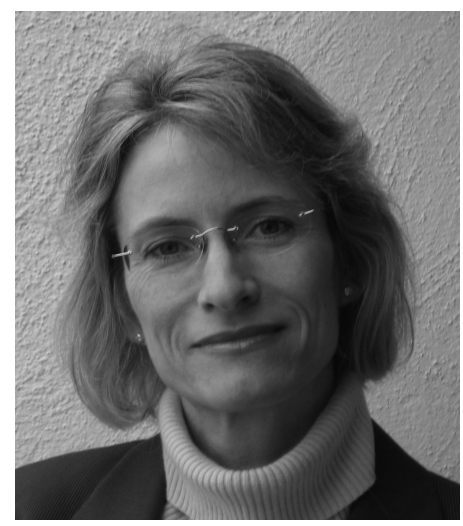

Tanja Scheer, Professorin für Alte Geschichte in Göttingen, 0. Mitglied der Akademie seit 2014
Die Akademie der Wissenschaften zu Göttingen tagt, um mit Hölderlin zu sprechen, traditionell „im Arm der Götter“ Griechenlands. ${ }^{1}$ Dies betrifft nicht nur den Sitzungssaal, von dessen Wänden Apollon und die neun Musen auf das Geschehen herabschauen. Es ist kein Zufall, dass die Große Aula des Universitätshauptgebäudes in Anlehnung an den Innenraum des Athener Parthenon gestaltet wurde: ein Altertumswissenschaftler und Mitglied der Akademie, Karl Otfried Müller, der von 1819 bis zu seinem frühen Tod 1839 in Göttingen wirkte, hat hierzu maßgeblich beigetragen. ${ }^{2}$

Das akademische Fach „Alte Geschichte“ erforscht in Deutschland schwerpunktmäßig die Geschichte der antiken Griechen und Rö-

mer, sowie deren Beziehungen zu ihren Nachbarkulturen. Die klassischen Kulturen der antiken Welt sind auch in der Gegenwart Europas hochgradig symbolisch aufgeladen. Dies zeigt sich etwa im Fall der aktuellen Krisenszenarien um das moderne Griechenland im Kontext der Europäischen Gemeinschaft. Griechenland ist nicht ein beliebiger dysfunktionaler Staat auf dem Balkan, sondern im historischen und rezeptionsgeschichtlichen Rückblick die Heimat einer Kultur, welcher wir einen Gutteil der Basis verdanken, auf der die Moderne Europas aufbaut. Schrift, Philosophie, Naturwissenschaft, Demokratie, aber auch der griechische Mythos, dessen Motive die europäische Kunst über Jahrhunderte geprägt haben, gehören zu diesem Erbe der antiken griechischen Kultur.

Die Antike ist präsent in unserer Lebenswelt - trotzdem werden die wenigsten Menschen Althistoriker. Die Aufgabe, sich selbst als neues Mitglied der Akademie der Wissenschaften zu Göttingen vorzustellen bietet Gelegenheit zur Reflexion

1 Friedrich Hölderlin, Da ich ein Knabe war.., in: F. Beissner (Hrsg.), Friedrich Hölderlin, Sämtliche Werke, Bd. 1.1, Stuttgart 1946, 267.

2 Fittschen, Klaus, Müller, Karl Otfried in: Neue Deutsche Biographie 18, 1997, 323-326 [Onlinefassung]; URL: http://www.deutsche-biographie.de/pnd11954153X.html; Christian Freigang, Die Baugeschichte, in: Marianne Bergmann, Christian Freigang u.a., Das Aula-Gebäude der Göttinger Universität. Athen im Königreich Hannover, München/Berlin 2006, 11-27. 
über die Herausbildung eigener Forschungsschwerpunkte und deren Verortung in der Fachkultur.

Interesse an althistorischen Themen kann durch ihre Vermittlung in Schule oder Universität geweckt werden. In den gedrängten Lehrplänen deutscher Gymnasien wird der Geschichte der antiken Welt allerdings nur wenig Raum zugestanden. Absolventen der gymnasialen Oberstufe sind bei einschlägigem Interesse meist gezwungen, den Umweg über die alten Sprachen, Latein und Griechisch, zu nehmen. In diesem Kontext kann die Leistung engagierter Lehrkräfte, die Schülern den Zugang zu den antiken Kulturen eröffnen und die notwendige intrinsische Motivation zur Weiterbeschäftigung mit diesem Thema hervorrufen, gar nicht bedeutsam genug eingeschätzt werden.

Mein Forschungsinteresse wurde allerdings nicht durch die Paideía ausgelöst, die das bayerische humanistische Gymnasium zu vermitteln suchte. Es entwickelte sich vielmehr in der unmittelbaren Begegnung mit den bildlichen und schriftlichen Zeugnissen des griechischen Mythos - also mit den zentralen Überlieferungen der griechischen Kultur, die von Göttern und Heroen handelten - und in der ebenso unmittelbaren Begegnung mit den Überresten dieser Kultur in ihrer Landschaft. In den frühen Siebziger Jahren des 20. Jh. war diese Unmittelbarkeit noch in anderer Weise gewährleistet als in der Gegenwart: die Cella des Parthenon auf der Athener Akropolis durfte etwa vom Besucher betreten werden, und der Tempel des Apollon Epikurios von Bassai - heute durch ein riesiges Zelt vor den Einflüssen der Witterung geschützt - stand frei sichtbar und frei zugänglich mitten in der Wildnis des arkadischen Berglands. Griechenland erwies sich jenseits der Großstädte als scheinbar intakte mediterrane Agrarkultur, die im positiven Sinne fremd erschien und auf manchmal überwältigender Gastfreundschaft beruhte. Die Wahrnehmung einer dörflichen Kultur in ihrem spezifischen Naturraum, in welchem der Fremde nicht bestohlen, sondern aus den eigenen beschränkten Ressourcen des bäuerlichen Haushalts beschenkt wurde, erwies sich für den Reisenden als prägende Erfahrung.

Drei hauptsächliche methodische Erkenntnisse wurzeln in den Erfahrungen dieser frühen Jahre; sie prägen meine Herangehensweise an wissenschaftliche Fragen noch heute: Aus den unzähligen Statuen, Vasenbildern und Bauwerken, die auf die griechischen Mythen Bezug nahmen, ergab sich, dass antike Texte und materiale Quellen untrennbar zusammengehörten. Und völlig selbstverständlich erschien darüber hinaus die Verbindung von Überlieferungstradition und Landschaft: mythische Erzählungen hatten konkrete Orte. Geburt und Wirken der griechischen Götter, die Taten und Tode der Heroen waren von den Menschen der Antike lokalisiert und in Tempeln, Naturheiligtümern und Monumenten erinnert und verehrt worden. Schließlich musste der fremde Naturraum die konkreten Lebensumstände der griechischen Kultur in jeder Hinsicht geprägt haben: dieser 
Naturraum war also - in seiner historischen Entwicklung - entsprechend zu berücksichtigen.

Welche Funktion hatte der Mythos in der griechischen Gesellschaft? Wie hingen Mythos und Religion zusammen? Wie wirkte sich der Naturraum sowohl auf die Erzähltraditionen als auch auf die konkrete Lebensweise der antiken Griechen aus?

Richtete man als Studienanfänger diese Fragen an die deutsche Alte Geschichte der frühen Achtziger Jahre des 20. Jahrhunderts - in München und darüber hinaus -, so stellte sich rasch heraus, dass die Spezialisten tendenziell andere Erkenntnisinteressen verfolgten.

Nach den Erfahrungen des Nationalsozialismus, während dessen Herrschaft sich auch Fachvertreter unrühmlich hervorgetan hatten, hatte sich ein großer Teil der deutschen Althistorie auf neopositivistische Positionen zurückgezogen. Die Rekonstruktion politischer Ereignisgeschichte unter dem Primat der Außenpolitik stand im Vordergrund. Ein Leittext dieser Ausrichtung war die „Griechische Geschichte“ von Hermann Bengtson, an prominenter Stelle im „Handbuch der Altertumswissenschaften" erschienen. ${ }^{3}$

Am Münchener Institut für Alte Geschichte hatte unter verschiedenen methodischen Ansätzen die Geschichte „Großer Männer“ Konjunktur: Christian Meier hatte soeben seine Biographie Caesars fertiggestellt, Jakob Seibert forschte über Alexander und Hannibal, mein späterer Lehrer Hatto H. Schmitt hatte sein wichtigstes Buch über den hellenistischen Herrscher Antiochos den Großen verfasst. ${ }^{4}$ Hermann Bengtson, in den frühen Achtziger Jahren des 20. Jhs. noch sehr aktiver Emeritus am Münchener Institut, hatte in seiner „Griechischen Geschichte“ kulturgeschichtliche Fragen in einem kurzen Kapitel unter dem Stichwort „Geistesgeschichte“ buchstäblich an den Rand gerückt. ${ }^{5}$ Darüber hinaus war er der dezidierten Meinung, Griechenland müsse man als Althistoriker nicht kennen. Diese Meinung vertrat er auch, nachdem das Reisen für deutsche Wissenschaftler nach dem zweiten Weltkrieg wieder problemlos möglich war. Der Verfasser der damals verbreitetsten „Griechischen Geschichte“ hatte die Akropolis nie gesehen.

3 Hermann Bengtson, Griechische Geschichte. Von den Anfängen bis in die Römische Kaiserzeit, Handbuch der Altertumswissenschaften III.4, München $1977^{5}$.

4 Christian Meier, Caesar, Berlin 1982; Jakob Seibert, Alexander der Große (EdF 10), Darmstadt 1972; Jakob Seibert, Hannibal, Darmstadt 1993; Hatto H. Schmitt, Untersuchungen zur Geschichte Antiochos’ des Großen und seiner Zeit (Historia Einzelschriften Bd. 6), Wiesbaden 1964.

5 Hermann Bengtson, Griechische Geschichte. Von den Anfängen bis in die Römische Kaiserzeit, Handbuch der Altertumswissenschaften III.4, München 19775 , 454-470: Die Weltherrschaft des griechischen Geistes. 
Von dieser Engführung hatten sich Bengtsons Schüler allerdings emanzipiert: sowohl Jakob Seibert als auch Bengtsons Nachfolger Hatto H. Schmitt, Münchener Ordinarius für Alte Geschichte, initiierten große Exkursionen in den Mittelmeerraum. ${ }^{6}$ Schmitt war auch offen gegenüber weiter gefassten Fragestellungen, die interdisziplinäre altertumswissenschaftliche Ansätze miteinschlossen. Diese inhaltliche Aufgeschlossenheit meines akademischen Lehrers manifestierte sich denn auch nicht nur in wohlwollender Duldung, sondern in steter aktiver Förderung meiner für das Fach seinerzeit eher ungewöhnlichen Interessenlagen. Dies machte wissenschaftliche Profilbildung unabhängig von fachspezifischen Schultraditionen möglich.

\section{Mythos}

Die Frage nach den Funktionen des Mythos in der griechischen Gesellschaft stand im Zentrum meiner Dissertation und erwies sich als ein Schlüssel zum Verständnis der Griechen und ihrer Nachbarn: ${ }^{7}$ Mythische Traditionen, so zeigte sich, waren nicht etwa „Märchen“ nur für Kinder oder „unwahr“, sie waren aber auch keine verbindlichen heiligen Texte. Antike Dichter mochten sich zuweilen auf göttliche Inspiration berufen, doch die griechischen Götter offenbarten keine „Heiligen Schriften“, die die Bürger der griechischen Polisstaaten hätten „glauben“ müssen. Keine zentrale sakrale Autorität wachte über dem griechischen Mythos, entsprechend gab es nicht die ursprünglich wahre Version, welche von vornherein uneingeschränkten Vorrang gegenüber späteren Traditionen genossen hätte. Gleichzeitig waren Mythen aber auch nicht von vornherein und per se unglaubhafte Erzählungen. Es handelte sich vielmehr „um den Komplex traditioneller Erzählungen, die man innerhalb einer bestimmten Kulturgruppe kennt“ und an dessen Kenntnis sich die Mitglieder der griechischen Gesellschaft als zugehörig erweisen konnten. ${ }^{8}$ Die Funktion griechischer mythischer Überlieferung hing entsprechend vom Kontext ab: Traditionen über die Taten der Götter

6 Das originellste derartige Unternehmen wurde von Jakob Seibert durchgeführt, den im Kontext seiner Forschungen zu Hannibal die Frage beschäftigte, auf welcher Route Hannibal die Alpen überquert hatte: auf den Spuren des karthagischen Feldherrn wanderte die Exkursionsmannschaft über verschiedene Alpenpässe: Jakob Seibert, Der Alpenübergang Hannibals. Ein gelöstes Problem?, Gymnasium 95, 1988, 21-73.

7 Tanja Susanne Scheer, Mythische Vorväter. Zur Bedeutung griechischer Heroenmythen im Selbstverständnis kleinasiatischer Städte (Münchener Arbeiten zur Alten Geschichte 7), München 1993.

8 Scheer, Mythische Vorväter, 16. 
machten deren Verhältnis untereinander und zu den Menschen deutlich - entsprechend waren sie von Bedeutung für religiöse Vorstellungen und erklärten mitunter als Aitiologien rituelles Handeln. Texte und Bilder, die die Taten der Heroen thematisierten, hatten nicht nur Unterhaltungswert, sondern sie erklärten Kultmale vor Ort, konstruierten mittels ausführlicher genealogischer Anbindung die Vergangenheit von Familien, Städten, Stämmen und Völkern und festigten so lokale und überregionale Identitäten. Mythische Tradition wurde so $\mathrm{zu}$ einem zentralen Bestandteil des antiken Bildes von der eigenen Frühgeschichte. Seit frühesten Zeiten war es möglich, Kritik an diesen Traditionen von Göttern und Heroen zu üben; welche Version im Einzelfall den höchsten Bekanntheitsgrad erreichte und als grundsätzlich glaubwürdig erachtet wurde, hing von Genre und historischem Kontext ab. Bereits antike Autoren des 6. Jh. v. Chr. wie z.B. Hekataios von Milet oder Xenophanes von Kolophon versuchten etwa durch Rationalisierung, durch Rückführung phantastischer Erzählelemente auf rational erklärbare Ursachen, glaubhafte und unglaubhafte Elemente mythologischer Erzählung zu scheiden. Ob die mythischen Traditionen der griechischen Kultur einen „historischen Kern“ in mehr oder weniger dunkler Frühzeit besitzen, also in Ereignisgeschichte früher Zeiten übersetzt werden können, und inwieweit solch postulierte „historische Erinnerung“ von modernen Historikern entschlüsselt werden kann, ist bis heute in der Forschung ein immer wieder neu diskutiertes Forschungsproblem, welches große methodische Schwierigkeiten aufwirft.

Im Unterschied dazu ging und geht es mir darum, die Funktionen mythischer Überlieferung in historisch helleren Epochen zu untersuchen: hierbei zeigt sich die immer wieder neue Aktualität des griechischen Mythos in der griechischen Geschichte: in unterschiedlichen Epochen - so stellte sich etwa heraus - versuchten Städte mitunter zweifelhafter Gräzität sich als Teil der griechischen Welt zu begreifen und dies auch nach außen zu tragen. Dies war möglich durch Vergangenheitskonstruktion: der Mythos erwies sich hierfür als interkulturelle Kommunikationsplattform. Zugehörig, verwandt und Freund war, wer z.B. Alexander dem Großen im 4. Jh. v. Chr. die richtigen Götter und Helden des Mythos als Stadtgründer und Urgroßväter plausibel machen konnte, indem es etwa gelang Orte des Mythos im eigenen Umland zu lokalisieren.

\section{Religion}

Aus der Untersuchung des Mythos in der griechischen Geschichte ergab sich die weiterführende Frage nach der Rolle und Bedeutung der Religion und religiöser Objekte in den griechischen Stadtstaaten. Auch diese Untersuchungsperspektive 
betraf auf einer Meta-Ebene die Frage, was denn nun die zentralen Themen der Alten Geschichte sein sollten. Ohne ihre Religion, die sich in Mythos und Kultpraxis manifestiert, sind die Griechen und ihr historisches Handeln nicht zu verstehen - inzwischen hat sich das Konzept griechischer Religion als eines Phänomens, das alle Bereiche sozialen und politischen Handelns durchdringt, auch in der althistorischen Forschung etabliert. Doch noch in den 90er Jahren des 20. Jahrhunderts kamen die Spezialisten für antike Religion überwiegend aus den Nachbarfächern, aus der Klassischen Philologie und der Klassischen Archäologie. ${ }^{9}$ Eine althistorische Perspektive auf „die Gottheit und ihr Bild“, die die Funktion dieser Götterbilder als Objekte in Religion und Politik in den Vordergrund stellte, erschien entsprechend eher ungewöhnlich. ${ }^{10}$ Mit dem Thema „Götterbilder“ hatten sich bisher vor allem Klassische Archäologen als ausgebildete Spezialisten für antike Plastik befasst, der Focus lag entsprechend tendenziell auf der Rekonstruktion, Datierung oder Zuordnung einzelner Statuen. Die Frage nach der religiösen oder gar gesamtgesellschaftlichen Funktion der Götterbilder wurde kaum gestellt. Wenn Fragen in diese Richtung zielten, dann erschienen zwei Kategorien als maßgeblich: ein Götterbild war entweder ein Kultbild (d.h. irgendwie religiös) oder ein Weihgeschenk (d.h. Dekoration). Aus dieser modernen Kategorisierung ergaben sich mitunter zweifelhafte Schlussfolgerungen: Auf der Athener Akropolis - um einen komplizierten Befund stark verkürzt zu beschreiben - standen sowohl im heiligen Bezirk als auch in verschiedenen sakralen Gebäuden mehrere Statuen der Athena. Sortierte man diese nun nach der Kategorie Kultbild oder Weihgeschenk, so nannten die antiken Quellen das Bild der Athena Polias im Erechtheion als das älteste Bild der Göttin. War dies nun das Kultbild Athens, mussten die anderen folglich Weihgeschenke sein. Weihgeschenke - das war die mehr oder weniger implizite Voraussetzung - hätten aber nur dekorativen Charakter, seien in religiöser Hinsicht nicht wichtig gewesen. Tempelbilder waren angeblich keine Weihgeschenke (dass sie normalerweise nicht vom Himmel fielen, sondern von Stiftern geweiht - also in Auftrag gegeben und finanziert worden waren, schien hierbei nicht aufzufallen). Als Schlussfolgerung wurde vielmehr das m.E. nicht

9 Nur einige Namen seien hier als prominente Beispiele genannt, die Klassischen Philologen Walter Burkert (Griechische Religion der archaischen und klassischen Epoche, erschienen erstmals Stuttgart 1977) und Fritz Graf (Nordionische Kulte. Religionsgeschichtliche und epigraphische Untersuchungen zu den Kulten von Chios, Erythrai, Klazomenai und Phokaia, Rom 1985), sowie die klassischen Archäologen Karl Schefold (Griechische Kunst als religiöses Phänomen, Hamburg 1961²) und Erika Simon (Die Götter der Griechen, München 1969).

10 Tanja Susanne Scheer, Die Gottheit und ihr Bild. Untersuchungen zur Funktion griechischer Kultbilder in Religion und Politik (Zetemata 105), München 2000. 
überzeugende Postulat vertreten, das zwölf Meter hohe Gold-Elfenbein-Bild der Athena im Parthenon - von Phidias geschaffen und neben dem Zeus von Olympia das berühmteste Götterbild der Antike - habe keine religiöse Bedeutung gehabt, sei „nur“ ein Weihgeschenk gewesen, bei dessen massiv goldenem Kleid sich der Athenische Staat habe nach Bedarf bedienen können. Da dieses Bild nur ein Goldspeicher gewesen sei, könne auch der Parthenon kein Tempel sein. Schlussfolgerungen wie diese erschienen zumindest fragwürdig. Ausgehend von der Analyse antiker Begrifflichkeit, in der sich eine derartige Trennung von Kultbildern und Weihgeschenken nicht abbildet, führte ein Perspektivenwechsel, der die Texte mit den materialen Quellen zur griechischen Religion in Verbindung setzte, zu einer anderen Annäherung an den Gegenstand. Zunächst einmal galt es nach dem überlieferten Umgang der Griechen mit den Bildern ihrer Götter in unterschiedlichen situativen Kontexten zu fragen: welches Verhalten gegenüber den Bildern erschien angemessen, von welchen Tabus waren diese Bilder umgeben, welche Folgen hatte der Verlust der Götterbilder etwa im Krieg? Hatten etwa die christlichen Autoren Recht, die behaupteten, die Heiden hielten die Bilder für die Götter?

Oder ließ sich hier eine chronologische Entwicklung erkennen, die religiösen Verfallshypothesen zur griechischen Geschichte entsprach? Glaubten die Griechen einer angeblich frommen Frühzeit die Götter seien mit ihren Bildern identisch, distanzierten sie sich in der Klassik - angeblich philosophisch aufgeklärt von dieser Vorstellung und straften dann im Hellenismus die Bilder der traditionellen Gottheiten mit Nichtachtung oder gar Zerstörung?

Die historische Analyse überlieferten Handelns im Umgang mit den Götterbildern konnte demgegenüber ein Erklärungsmodell wahrscheinlich machen, das Götterbilder als Medien zur Schaffung potentieller und zeitweiliger göttlicher Präsenz verstand: Götterbilder konnten für die Griechen im Wortsinn „hedos“, „Sitz“ der Gottheit sein. Sie waren ein Mittel um potentielle göttliche Anwesenheit zu fördern. Ob sich der Einzelne vorstellte, die Gottheit habe zeitweilig unmittelbar im Bild Platz genommen oder halte sich in seiner Nähe auf, lässt sich aus den Quellen nicht als allgemeingültige Aussage ableiten. Einzelne Bewohner und Bewohnerinnen einer griechischen Polis waren nicht auf autoritär formulierte Glaubenssätze verpflichtet. Die Normen der Polis betrafen den konkreten Umgang mit den Bildern der Götter, die man nicht berauben oder beschmutzen durfte oder zu denen der Zugang nicht immer gestattet war. Die Bedeutung einzelner Götterbilder war nicht normativ festgelegt, sondern ergab sich aus der als traditionell verstandenen, faktisch aber veränderlichen kultischen Praxis. „Ein Kultbild“, so hieß es im Resümee meiner Habilitationsschrift, „entsteht dann und dort, wo ein Einzelner oder eine Gruppe versucht, vor einer Götterstatue mit der Gottheit Kontakt aufzunehmen und sie zum Platznehmen zu bewegen. So ist das Kultbild 
der Gottheit keine absolut gesetzte Größe. Es entsteht immer neu in der Verehrung durch sein menschliches Gegenüber. “11

\section{Gender}

Erwiesen sich Mythos und Religion als Themen, deren Behandlung von Seiten der Alten Geschichte nicht selbstverständlich war, lag ein drittes Forschungsthema aufgrund der gesellschaftlichen Umbrüche seit den späten 60er Jahren des 20. Jh. zwar schon seit geraumer Zeit in der Luft, galt aber de facto ebenfalls nicht als zentrales Thema der Alten Geschichte. Dies war das Thema „Gender“. Auch die griechische Kultur und Geschichte waren von Männern und Frauen gleichermaßen als historisch Handelnden getragen worden. Dies erscheint zunächst als Gemeinplatz. In den deutschsprachigen Altertumswissenschaften um die Jahrtausendwende hatte sich allerdings die Erkenntnis, dass die Perspektive „Geschlecht“ im Hinblick auf alle Bereiche der antiken Welt erweiterte historische Erkenntnisse generieren konnte, noch nicht auf breiter Front durchgesetzt. Als die Herausgeber der Zeitschrift Gymnasium Ende der neunziger Jahre mit dem freundlichen Angebot an mich herantraten, in ihrer Reihe „Forschungsüberblicke“ einen solchen Überblick zum vorformulierten Thema „Forschungen über die Frau in der Antike“ zu verfassen, ${ }^{12}$ verdankte ich diese Ehre wohl nicht zuletzt der Tatsache, selbst dem weiblichen Geschlecht anzugehören, welches in der universitären Alten Geschichte nur als eine verschwindend kleine Minderheit sichtbar war. ${ }^{13}$ Die folgende vertiefte Beschäftigung mit diesem neuen Thema führte zwangsläufig sehr rasch zur Erkenntnis seiner inhaltlichen und akademischen Brisanz.

Die Frage nach den Geschlechterverhältnissen in der griechischen Antike erweist sich sowohl für kulturgeschichtliche Fragestellungen als auch für die Wahrnehmung moderner sozialer Kontexte als konstruktive und weiterführende Perspektive: Nicht inwieweit und in welcher Form griechische Männer über ihre Frauen herrschten, ist hierbei die zentrale Frage: es geht vielmehr darum, für die Männer und die Frauen der griechischen Antike die Kategorie Geschlecht und ihre

11 Scheer, Die Gottheit und ihr Bild, 305.

12 Tanja S. Scheer, Forschungen über die Frau in der Antike. Ziele, Methoden, Perspektiven, Gymnasium 107, 2000, 143-172.

13 Im Hinblick auf die Bedeutung, die die neuere Bildungsforschung sogenannten, role models‘ zuschreibt, sei ergänzend hinzugefügt: zu Beginn meines Studiums im Jahr 1983 gab es in Deutschland keine Frau auf einem althistorischen Lehrstuhl, von den sehr wenigen Professorinnen für Alte Geschichte lehrte keine im Bundesland Bayern. 
Auswirkungen in ganz unterschiedlichen Bereichen historischer Forschung zu berücksichtigen. ${ }^{14}$ Antike Geschlechterbilder - und dieser Aspekt erscheint zusätzlich hochinteressant - haben außerdem ihre Wirkungskraft nicht mit dem Ende der Antike verloren. Vermittelt durch mythologische, medizinische und philosophische Konzepte, die mit der Autorität antiker Protagonisten mit seinerzeit wissenschaftlichem Anspruch verbunden werden, können sie bis heute wirksam sein. Hippokrates, Platon und Aristoteles haben auch neuzeitliche Vorstellungen vom richtigen Verhältnis der Geschlechter geprägt.

Mythos, Religion und Gender sind in der Vergangenheit der akademischen Alten Geschichte streckenweise nicht berücksichtigt oder vom angeblich eigentlichen geschichtlichen Handeln abgegrenzt worden. Nutzt man sie aber als Untersuchungskategorien, als Perspektiven auf das scheinbar vertraute Material, so lässt sich dieses in neuer Weise fruchtbar machen. Ungewöhnliche Perspektiven können dann in überraschende, inhaltlich und chronologisch breit gefächerte Kontexte führen: mythische Traditionen, die z.B. vom griechischen Heros Herakles berichten, können sich etwa als vom Alten Orient beeinflusst erweisen; für das Athen der archaischen Zeit sind sie als mögliche Ausdrucksmittel für Tyrannenherrschaft diskutiert, für Sparta als Argumente früher Landnahme herangezogen worden. Im Hellenismus dient Herakles als Beleg für Verwandtschaft mit Alexander dem Großen, während sich im Panhellenion Kaiser Hadrians mit dem mythischen Vorvater Herakles der Beweis für griechische Abkunft und kulturelle Zugehörigkeit erbringen lässt. Dass die Verehrung des Herakles sowohl als Heros als auch als Gott zahlreiche Fragen für den Religionshistoriker aufwirft, dürfte ebenso einsichtig sein, wie auch seine Bedeutung für die Konstruktionen von Männlichkeit in der griechischen und römischen Gesellschaft.

\section{Wie geht es weiter? Mythos, Gender, Religion in der Gegenwart der Alten Geschichte in Göttingen}

Übergreifende Perspektiven wie Mythos, Religion, Gender erweisen sich in der akademischen Gegenwart des Faches Alte Geschichte als vielfach anschlussfähig. Freilich bedarf es hierzu eines akademischen Umfeldes, in dem die altertumswissenschaftlichen Nachbardisziplinen in möglichster Breite vorhanden sind, so dass interdisziplinäre Zusammenarbeit auch fachspezifischen Mehrwert für alle Beteiligten ergibt. Der Standort Göttingen bietet diese Möglichkeiten grundsätz-

14 Tanja Susanne Scheer, Griechische Geschlechtergeschichte (Enzyklopädie der griechischrömischen Antike 11), München 2011. 
lich seit jeher: als besonders ertragreich für die Definition und Entwicklung gemeinsamer Forschungsinteressen hat sich in den letzten Jahren die Verbindung zahlreicher altertumswissenschaftlicher und theologischer Fächer im Centrum Orbis Orientalis et Occidentalis (CORO = Zentrum für Antike und Orient) erwiesen. Die Akademie der Wissenschaften zu Göttingen zählt bezeichnenderweise zu den Trägern dieser wichtigen Kommunikationsplattform.

Nicht zuletzt auf der Basis des CORO hat die Alte Geschichte in Göttingen ihre Forschungsperspektiven in den letzten Jahren in eine Reihe erfolgreicher Einzelund Verbundforschungsprojekte einbringen können: Mythos und Landschaft stehen etwa im Zentrum eines althistorischen DFG- Projekts zum Thema „Wo liegt Arkadien? Arkadienbilder in der klassischen Antike““. ${ }^{15}$ Erforscht wird hier die Konstruktion einer bis in die Gegenwart „legendären“ antiken Landschaft vor allem in der mythischen Tradition Griechenlands und Roms.

Mit den Schwerpunkten mythische Tradition, Religion und Gender bringt sich die Alte Geschichte im neuen DFG-Sonderforschungsbereich „Bildung und Religion in Kulturen des Mittelmeerraums und ihrer Umwelt von der Antike bis zum Mittelalter und zum klassischen Islam“ ein: unter der Überschrift „Aufgeklärte Männer - abergläubische Frauen? Religion, Bildung und Geschlechterstereotypen im klassischen Athen“ wird nach der Vermittlung des „richtigen“ und der Definition des „falschen“ religiösen Wissens in der polytheistisch geprägten griechischen Polis des 5. und 4. Jahrhunderts v. Chr. gefragt. ${ }^{16}$

Die inhaltliche Entwicklung und Veränderung griechischer Mythen im sozialen und politischen Kontext wird schließlich in den kommenden Jahren einen weiteren wichtigen Forschungsschwerpunkt der Alten Geschichte in Göttingen bilden: Die Aufgabe, Mythen in interdisziplinärer Perspektive zu untersuchen, steht im Zentrum der neuen DFG-Forschergruppe „STRATA. Stratifikationsanalysen mythischer Stoffe und Texte in der Antike“. In diesem Forschungsverbund werden Altorientalistik, Klassische Philologie, Altes und Neues Testament, Kirchengeschichte und Alte Geschichte zusammenarbeiten. ${ }^{17}$ Mythos, Religion und Gender - das zeigt diese Auswahl drittmittelfinanzierter Forschungsprojekte sind ganz offensichtlich im Zentrum der Altertumswissenschaften angekommen und die Alte Geschichte kann hierzu in verschiedenen Kontexten und unter verschiedenen Fragestellungen ihren Beitrag leisten.

Für das Fach Alte Geschichte zur Zeit meines Studienbeginns war der griechische Mythos bestenfalls ein Nebenschauplatz. Als wissenschaftlicher Anfän-

15 DFG-Projekt Nummer: GZ SCHE 321/4-1.

16 SFB 1136, Federführung Peter Gemeinhardt (Kirchengeschichte und Patristik).

17 FOR 2064 Federführung Annette Zgoll (Altorientalistik). 
ger musste man die Fachwelt von der Wichtigkeit dieses Themenfeldes erst einmal überzeugen. Der Kampf für den Mythos hat jedoch Tradition, und hierbei spielen die Georg-August-Universität und die Akademie eine besondere Rolle. Die Akademie der Wissenschaften zu Göttingen zählt einen besonders bedeutenden Gelehrten zu ihren „Mythischen Vorvätern“, an den ich in diesem Zusammenhang erinnern möchte: fast ein halbes Jahrhundert ist er als Generalsekretär der Akademie das Herz dieser Institution gewesen und er begriff es als Lebensaufgabe, den griechischen Mythos als wichtigen Forschungsgegenstand zu etablieren. Die Rede ist von Christian Gottlob Heyne (1729-1812). Heyne hielt seine erste von insgesamt 17 mythologischen Vorlesungen vor der Akademie im Jahr 1763 zum Thema „Die Erinnerung an die mythischen Zeiten“. Er musste sich noch gegen geläufige Ansätze wehren, die die griechische Mythologie vollständig aus dem Alten Testament ableiten wollten. ${ }^{18}$ Heute arbeiten in Göttingen klassische Altertumswissenschaftler und Theologen zum Thema Mythos einträchtig zusammen. Als Althistorikerin im 21. Jh. mit den Forschungsschwerpunkten Mythos und Religion in die Akademie Heynes aufgenommen worden zu sein, lässt mich in besonderem Maße hoffen, diese Themenfelder als Perspektiven auf die Alte Welt im kollegialen Miteinander der Akademie weiter zu vertiefen.

18 Tanja Susanne Scheer, Heyne und der griechische Mythos, in: Balbina Bäbler/Heinz-Günther Nesselrath, Christian Gottlob Heyne, Werk und Leistung nach zweihundert Jahren (Abhandlungen der Akademie der Wissenschaften zu Göttingen N.F. 32), Berlin/New York 2014, 1-28, 6 f., 12. 